\title{
Concentración de mucina salival en pacientes con enfermedad periodontal
}

\author{
Salivary mucin concentration in patients with periodontal disease \\ Concentração de mucina salivar em pacientes com doença periodontal
}

\author{
Miguel Jorge Acuña ${ }^{1}$ (iD) 0000-0002-3520-2425 \\ Rolando Pablo Juárez² (D) 0000-0001-8950-3373
}

\section{Resumen}

El objetivo de este trabajo fue estudiar la relación entre la concentración de mucina salival y la enfermedad periodontal.

La muestra se dividió en tres grupos de 20 individuos cada uno: Grupo 1 sin enfermedad periodontal; Grupo 2 con gingivitis; y Grupo 3 con periodontitis.

En todas las muestras salivales se confirmó la presencia de mucina, el Grupo 1 presentó un valor promedio de 1,27 mg/ml. En el Grupo 2 se registró un promedio de 1,93 mg/ml. En el Grupo 3 se observó un promedio de 3,01 mg/ml.

El Análisis de la Variancia y posterior prueba de $\mathrm{F}(\mathrm{F}=25,01, \mathrm{p}<0,0001)$ confirman diferencias significativas en los contenidos de mucina entre grupos.

El aumento de la concentración de mucina salival en pacientes periodontales podría representar un marcador químico de utilidad como coadyuvante en el diagnóstico clínico de esta enfermedad.

Palabras clave: saliva-mucina-gingivitis-periodontitis.

Cátedra Física Química Biológica -Facultad Odontología, UNNE, Argentina, odontoacuna@gmail.com

Cátedra Fisiología Humana, Facultad Odontología, UNNE, Argentina 


\begin{abstract}
The objective of this work was to study the relationship between salivary mucin concentration and periodontal disease.

The sample was divided into three groups of 20 individuals each: Group 1 without periodontal disease; Group 2 with gingivitis; and Group 3 with periodontitis.

In all salivary samples the presence of mucin was confirmed, Group 1 presented an average value of $1.27 \mathrm{mg} / \mathrm{ml}$. In Group 2 an average of $1.93 \mathrm{mg} / \mathrm{ml}$ was recorded. In Group 3 an average of $3.01 \mathrm{mg} / \mathrm{ml}$ was observed.

The Analysis of Variance and subsequent $F$ test $(\mathrm{F}=25.01, \mathrm{p}<0.0001)$ confirm significant differences in mucin contents between groups.

Increased salivary mucin concentration in periodontal patients could represent a useful chemical marker as an adjunct in the clinical diagnosis of this disease.
\end{abstract}

Keywords: saliva-mucin-gingivitis-periodontitis.

\section{Introducción}

El termino enfermedad periodontal (EP) engloba un amplio grupo de enfermedades infecciosas, inflamatorias, trastornos metabólicos y neoplasias que pueden afectar la encía, los tejidos conectivos periodontales y el hueso alveolar. Desde el punto de vista clínico, se la puede clasificar en gingivitis (cuando la lesión afecta la encía) y periodontitis (cuando la lesión afecta el ligamento periodontal, el cemento y el hueso alveolar), provocando zonas de inflamación y destrucción tisular a nivel local ${ }^{(1)}$. Epidemiológicamente es la segunda patología de mayor prevalencia en la cavidad bucal en todo el mundo ${ }^{(2)}$, uno de los factores más predisponentes para la formación de esta enfermedad es la pre-

\section{Resumo}

O objetivo deste trabalho foi estudar a relação entre a concentração de mucina salivar e a doença periodontal.

A amostra foi dividida em três grupos de 20 indivíduos cada: Grupo 1 sem doença periodontal; Grupo 2 com gengivite; e Grupo 3 com periodontite.

Em todas as amostras salivares foi confirmada a presença de mucina, o Grupo 1 apresentou valor médio de $1,27 \mathrm{mg} / \mathrm{ml}$. No Grupo 2, foi registrada uma média de 1,93 mg / ml. No Grupo 3 foi observada uma média de 3,01 mg / ml.

A Análise de Variância e o teste F subsequente $(\mathrm{F}=25,01, \mathrm{p}<0,0001)$ confirmam diferenças significativas nos conteúdos de mucina entre os grupos.

O aumento da concentração de mucina salivar em pacientes periodontais pode representar um marcador químico útil como adjuvante no diagnóstico clínico desta doença.

Palavras-chave: saliva-mucina-gengiviteperiodontite.

sencia de bacterias patógenas que residen en el biofilm ${ }^{(3)}$. En la actualidad los medios de diagnósticos utilizados para confirmar o descartar la presencia de esta patología solo denotan la destrucción tisular provocada, pero no determinan si esa enfermedad está en reposo o activa. Lo que hace difícil para el odontólogo clínico realizar un tratamiento correcto ${ }^{(4)}$.

La saliva que es una secreción de fácil acceso, no invasiva y poco traumática para el paciente a la hora de tomar una muestra para ser analizada. Existen elementos presentes en la saliva que pueden ser utilizados como marcadores bioquímicos de la enfermedad periodontal, estos nos pueden ayudar a determinar si la patología está en actividad o reposo, como así también si está en su etapa inicial, estos elementos pueden ser proteínas, 
enzimas como por ejemplo la fosfatasa alcalina salival, amilasa salival, entre otras ${ }^{(5-6-7)}$.

Otro marcador químico que se podría utilizar es la mucina salival, una proteína presente en la saliva y desarrolla varias funciones a nivel bucal, una de ellas es proteger al organismo de los microorganismos invasores uniéndose a ellos y aglutinándolos, debido a esta característica, creemos que puede estar elevada su concentración en la enfermedad periodontal ${ }^{\text {(8-9-10-11-12-13). }}$. El objetivo de este trabajo fue estudiar la relación entre la concentración de mucina salival y la enfermedad periodontal.

\section{Materiales y métodos}

Para este estudio se trabajó con pacientes en un rango de 18 a 60 años y de ambos sexos que concurrieron a la Facultad de Odontología dependiente de la Universidad Nacional del Nordeste (UNNE) - Argentina. Entre ellos se seleccionaron para la muestra los que concurrieron con diagnóstico de EP definido en base a parámetros clínicos (índice gingival GI - sangrado al sondaje - profundidad de bolsa BOP y pérdida de inserción-PI) y a los hallazgos radiográficos y que participen voluntariamente en el estudio con firma de consentimiento informado. Se excluyeron de la muestra embarazadas, pacientes que hubieran recibido tratamiento periodontal previo o utilizado antibióticos u otra medicación durante los seis meses anteriores a la consulta, que presentaban enfermedades crónicas como diabetes o hipertensión arterial, problemas psicológicos o capacidades cognitivas disminuidas y pacientes que no prestaron su consentimiento para participar del estudio, aquellos.

Posterior a la evaluación clínica periodontal, los pacientes de la muestra se dividieron en tres grupos de 20 individuos: Grupo 1 (control), pacientes con diagnóstico negativo de enfermedad periodontal; Grupo 2, pacientes con gingivitis; y Grupo 3 pacientes con periodontitis. Luego se procedió a la toma de muestras salivales para determinar la concentración de mucina en cada paciente. Todas las muestras de saliva se recogieron en un rango horario comprendido entre 8:00 am y 10:00 am, utilizando una técnica de recolección de saliva (no estimulada) ${ }^{(14)}$.

Las muestras de saliva se recolectaron en una sesión por cada individuo en horario prefijado, se utilizaron recipientes de propileno desechables, estériles, con tapa enroscable, de $15 \mathrm{ml}$ de capacidad y luego se colocaron en tubos de ensayo. Se añadió posteriormente $1 \%$ de solución de azul Alcian en $50 \mathrm{ml}$ de acetato de sodio (buffer) y se incubó a temperatura ambiente bajo agitación continua durante 30 minutos. Después de la incubación, las muestras se centrifugaron durante $20 \mathrm{~min}$ a $3000 \mathrm{rpm}$, se añadió $1 \mathrm{ml}$ de etanol al $95 \%$ y se agitó durante 10 seg. Después de $5 \mathrm{~min}$, las muestras fueron centrifugadas durante $20 \mathrm{~min}$ a $3000 \mathrm{rpm}$, posteriormente se colocó Aerosol OT (diluciones 1:2 con agua destilada) seguido por una adición de igual cantidad de éter etílico con agitación vigorosa. Las muestras se centrifugaron durante 15 min a $3000 \mathrm{rpm}$ y se midió la densidad óptica a $605 \mathrm{~nm}$ utilizando un espectrofotómetro UV. A partir de esta lectura se calculó la concentración de mucina utilizando un gráfico estándar (15). Todas las determinaciones se realizaron en el Área Fisicoquímica, del Laboratorio de Investigaciones Científicas de la Facultad de Odontología de la UNNE.

Se registró, además el género ( $\mathrm{F}$ y $\mathrm{M}$ ) y la edad de cada paciente en años, la que fue posteriormente dividida en tres categorías (E1: de 18 a 33 años, E1 de 34 a 46 años y E3: de 47 a 60 años).

La información fue volcada en una planilla de datos Excel y luego analizada mediante el Software Estadístico InfoStat $2020^{(16)}$. A los fines de analizar el comportamiento general de la información, se realizó un análisis exploratorio de los datos, de manera gráfica y analítica. Las asociaciones entre variables y su significancia ( $\square=0,05)$ se estudiaron, a través del Coeficiente de Correlación de Pearson para las variables cuantitativas y por Pruebas de Independencia 
mediante el estadístico Chi-Cuadrado para las variables cualitativas. Con la finalidad de establecer diferencias significativas $(\square=0,05)$ en los valores de mucina entre los diferentes grupos, se realizaron Análisis de la Variancia y pruebas F, con posteriores pruebas de Duncan a fin de la separación de las medias de grupos ${ }^{(17)}$.

\section{Resultados}

La muestra estuvo constituida por un $50 \%$ de varones y un $50 \%$ de mujeres. Las edades oscilaron entre los 18 y los 60 años, en la Figura 1 se presentan las distribuciones de edades en cada uno de los grupos en estudio, de donde se puede determinar la equivalencia de las muestras. La prueba de Chi cuadrado arrojó un valor $\square^{2}=0,19$ ( $\left.\mathrm{p}=0,9958\right)$ lo que indica homogeneidad en la distribución de las categorías de edad entre grupos.

Figura 1: Distribuciones de edades de los pacientes en los tres grupos estudiados

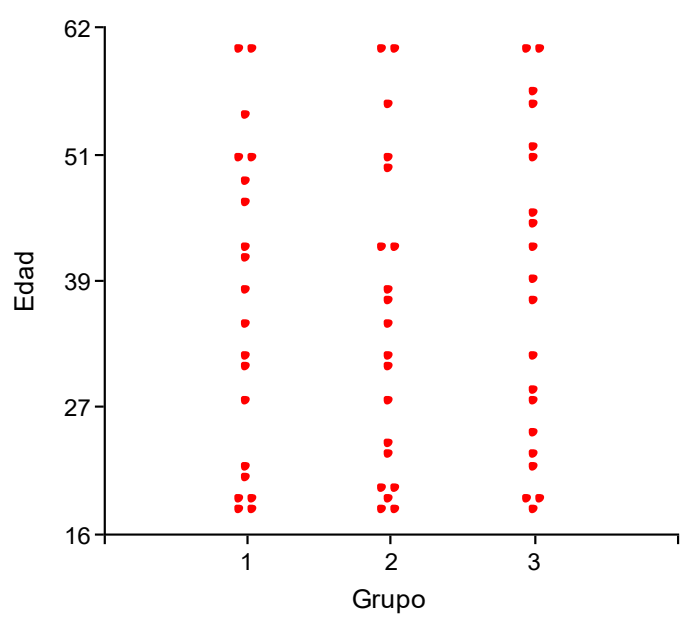

En todos los pacientes de la muestra se confirmó la presencia de mucina, el Grupo 1 presentó un valor mínimo de mucina salival de $0,08 \mathrm{mg} /$ $\mathrm{ml}$ y un máximo de $2,48 \mathrm{mg} / \mathrm{ml}$, un promedio de $1,27 \mathrm{mg} / \mathrm{ml}$, con una desviación estándar de $0,63 \mathrm{mg} / \mathrm{ml}$. En el Grupo 2 se registró un mínimo de $1,20 \mathrm{mg} / \mathrm{ml}$ y un máximo de 3,70 $\mathrm{mg} / \mathrm{ml}$, un promedio de $1,93 \mathrm{mg} / \mathrm{ml}$ y una des- viación estándar de 0,61 mg/ml. En el Grupo 3 se observó un mínimo de $1,40 \mathrm{mg} / \mathrm{ml}$ y un máximo de $4,89 \mathrm{mg} / \mathrm{ml}$, un promedio de 3,01 $\mathrm{mg} / \mathrm{ml}$ y una desviación estándar de $1,03 \mathrm{mg} /$ $\mathrm{ml}$. En la Figura 2 se presentan los contenidos promedio de mucina y sus errores estándares por grupo de estudio.

Figura 2: Promedios y errores estándares del contenido de mucina en saliva en los tres grupos en estudio

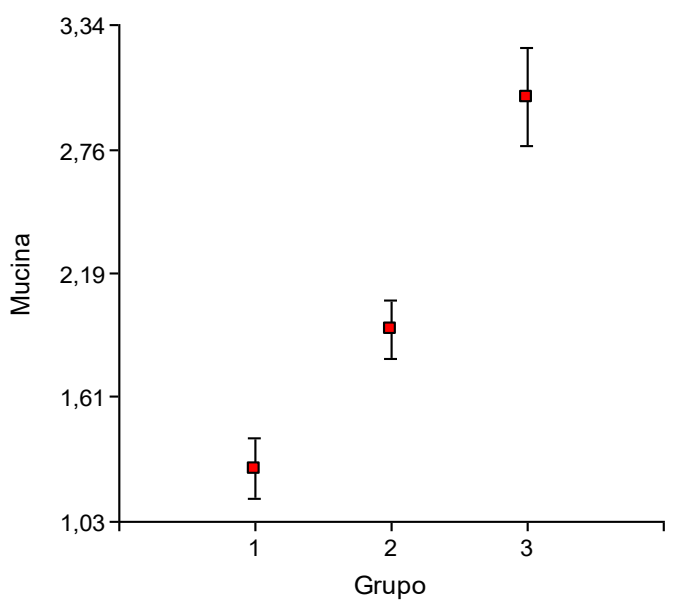

El Análisis de la Variancia y posterior prueba de $\mathrm{F}(\mathrm{F}=25,01, \mathrm{p}<0,0001)$ confirman diferencias significativas en los contenidos de mucina entre grupos, los resultados de la prueba de Duncan que se presentan en la Tabla 1, permiten afirmar que los contenidos medios de mucina en el Grupo 1 son inferiores a los de los otros dos grupos y que los del Grupo 2 a su vez son inferiores a los del Grupo 3.

Tabla 1: Promedios de contenidos de mucina por grupo estudiado y diferencias según prueba de Duncan

\begin{tabular}{|c|c|c|c|c|}
\hline Grupo & Me & & & \\
\hline 1 & 1,27 & A & & \\
\hline 2 & 1,93 & & B & \\
\hline 3 & 3,01 & & & C \\
\hline
\end{tabular}

Medias con una letra común no son significativamente diferentes ( $\mathrm{p}>0,05)$ 
En la Figura 3 se presentan los promedios y errores estándares de los contenidos de mucina por grupo de estudio y por género. Si bien el incremento en el contenido de mucina que se observa al pasar del Grupo 1 al 2 y de éste al 3 pareciera ser más marcado en los hombres que en las mujeres, la prueba de $\mathrm{F}(\mathrm{F}=3,1$, $\mathrm{p}=0,0856)$ no detectó efecto significativo del género.

Figura 3: Promedios y errores estándares del contenido de mucina en saliva en los tres grupos en estudio, por género
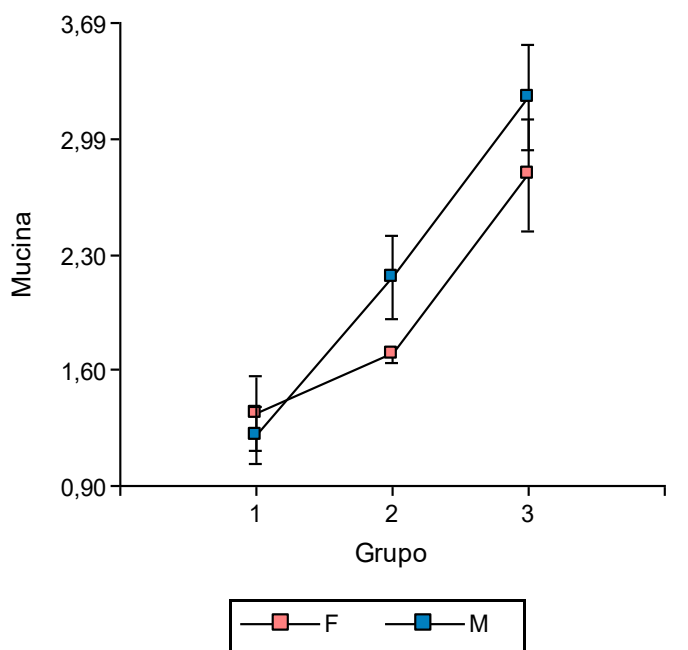

Al estudiar la asociación entre el contenido de mucina y la edad, los coeficientes de correlación de Pearson no resultaron significativos en los Grupos $1(\mathrm{r}=-0,13, \mathrm{p}=0,5834)$ y $3(\mathrm{r}=0,06$, $\mathrm{p}=0,8135)$, pero si en el Grupo $2(\mathrm{r}=0,60$, $\mathrm{p}=0,005)$, lo que indica que en los pacientes con periodontitis al aumentar la edad se incrementa el contenido de mucina en saliva. En la Figura 4 se pueden observar los contenidos medios de mucina en saliva por grupo de estudio y por categoría de edad.
Figura 4: Contenidos medios de mucina en saliva y errores estándar, por grupo de estudio y por categoría de edad

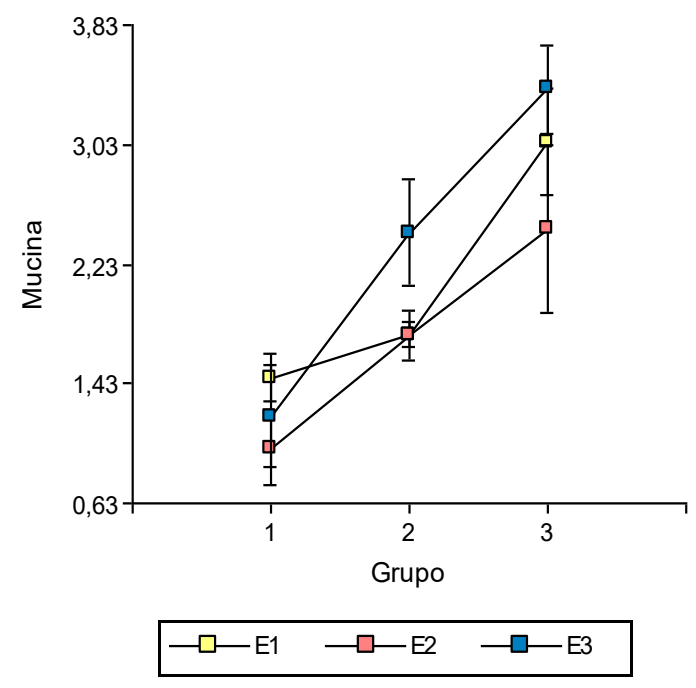

\section{Discusión}

No obstante, el avance de estos medios de diagnósticos todavía no se ha logrado predecir cómo será la destrucción periodontal en cada individuo. Es importante la detección clínica precoz de la enfermedad en los pacientes con lesiones iniciales a través de algún indicador que resulte de fácil acceso y lectura y que permita detectar prematuramente zonas afectadas o casos de riesgo, para implementar estrategias adecuadas de planeamiento y/o de prevención.

Al producirse una actividad eminentemente inflamatoria deberían contarse con marcadores químicos que permitan detectar la posible agresión a los tejidos. Por cuya razón, precisamente a nivel local deberían estudiarse algunas de las sustancias presentes en la saliva como la mucina. En el presente artículo se determinó la actividad de la mucina salival como biomarcador en pacientes con enfermedad periodontal. En este estudio se mostró un marcado aumento de concentración de mucina en la saliva de pacientes con gingivitis y más aún con periodontitis.

La concentración de mucina normal en saliva es de $1,19 \mathrm{mg} / \mathrm{ml}$, Swati Kejriwal y cols. ${ }^{(15)}$, demostraron un aumento en la concentración de 
mucina salival, en pacientes con gingivitis, y un descenso en la concentración de mucina en el grupo de periodontitis en comparación con el grupo de gingivitis. Coincidiendo con dicho estudio, los resultados de este trabajo manifestaron un marcado aumento de la concentración de mucina en saliva de pacientes con gingivitis, pero en contraposición a lo hallado por estos autores, los pacientes con periodontitis presentaron una concentración de mucina en saliva mayor que los pacientes con gingivitis.

Sánchez GA y cols. ${ }^{(18)}$ determinaron que las concentraciones de proteínas, amilasa y mucina aumentaron en sujetos con enfermedad periodontal moderada y severa en saliva no estimulada, similar resultado se obtuvo en este trabajo en cuanto al aumento de la concentración de mucina en pacientes con periodontitis.

Andrea Beatriz Acquier y cols. ${ }^{(19)}$ describieron en su trabajo que la concentración de mucina salival aumenta en pacientes con periodontitis crónica y periodontitis agresiva, este estudio coincide en cuanto al aumento de la concentración de mucina salival en los pacientes con periodontitis.

Lundmark A. y cols. ${ }^{(20)}$ determinaron una disminución de la concentración de mucina salival en pacientes con periodontitis, contrariamen- te a lo encontrado en este trabajo en donde la concentración de mucina en pacientes con periodontitis es la más elevada de los tres grupos estudiados.

\section{Conclusiones}

El aumento de la concentración de mucina en saliva de pacientes periodontales podría representar un marcador químico de utilidad como coadyudante en el diagnóstico clínico de esta enfermedad.

Existe una relación directa entre el contenido de mucina en saliva y la enfermedad periodontal. La concentración de mucina salival es menor en pacientes con diagnóstico negativo de enfermedad periodontal, se incrementa en pacientes con gingivitis y más aún en pacientes con periodontitis.

No existe efecto de género sobre el contenido de mucina salival en los grupos estudiados.

El contenido de mucina salival y la edad del paciente no se correlacionan significativamente en pacientes con gingivitis o diagnóstico negativo de enfermedad periodontal. En los pacientes con periodontitis el contenido de mucina en saliva se incrementa con la edad.

\section{Referencias}

1. Herrera D, Figuero E, Shapira L, Jin L, Sanz M. La Nueva clasificación de las enfermedades periodontales y periimplantarias. Rev Cient Soc Esp Period. 2018;1(9):94-110.

2. Martínez LL, López MDCGC, Rodríguez AWH, Sierra EMS, Luna MAL, Rodríguez SHS. La caries, gingivitis, periodontitis y la maloclusión siguen siendo las afecciones estomatológicas más frecuentes en la población. Arch Med. 2014;9(4).

3. Lindhe J, Karring T, Lang NP. Periodontología clínica e implantología odontológica. 5 ed. Educ Med Panamericana; 2009289 p.

4. Newman MG, Takei H, Klokkevold PR, Carranza FA. Carranza's clinical periodontology. 11 ed. Elsevier health sciences; $2011176 \mathrm{p}$.

5. Romero C, Suarez M, Gloria-Narváez C. Proteínas Totales, Fosfatasa Alcalina, Prostaglandinas E2 y Lisozima como Biomarcadores Salivales en Pacientes Adultos con Periodontitis Crónica. Int J Odontostomatol. 2017;11(4):381-5.

6. Villacís Tapia ÁF 2017. Niveles de la enzima alanino aminotransferasa en saliva en periodontitis crónica y su relación con la severidad (Master's thesis, Quito: UCE). 
7. Baudo JE, Tosti SB, Mazzeo DMA, Cecho AC, Allegretti PE. IL-1 y TNF $\alpha$ como biomarcadores salivares de enfermedad periodontal. Rev Fac Odontol. 2015;2015.

8. Johansson MEV, Hansson GC. 2016. The mucins. Encyclopedia of immunobiology. P. 381-8.

9. Hernández Castañeda AA, Aranzazu Moya GC. Características y propiedades físico-químicas de la saliva: una revisión. UstaSalud. 2012;11(2):102-12. doi: 10.15332/us.v11i2.1123.

10. Gésime JM, Acevedo AM, Lalaguna F. Las mucinas salivales y sus implicaciones en la reología de la saliva humana y los sustitutos salivales. Acta Odontol Venez. 2009;47(2):446-52.

11. Dhanisha SS, Guruvayoorappan C, Drishya S, Abeesh P. Mucins: structural diversity, biosynthesis, its role in pathogenesis and as possible therapeutic targets. Crit Rev Oncol Hematol. 2018; 122:98-122. doi: 10.1016/j.critrevonc.2017.12.006

12. Diajil AR, Sood LI, Azeez RA. A Salivary $\alpha$-amylase Level in Relation to the Oral Health Parameters among Children in Baghdad City. Journal of baghdad college of dentistry. 2016;28(2):40-6.

13. Daza ANZ, Huerta ERL, Martínez FF. Determinación de $\mathrm{pH}$ y proteínas totales en saliva en pacientes con y sin aparatología ortodóncica fija (estudio piloto). Rev Odontol Mex. 2004;8(3):59-63.

14. Slomiany BL, Murty VLN, Piotrowski J, Slomiany A. Salivary mucins in oral mucosal defense. Gen Pharmacol Vasc Syst. 1996;27(5):761-71. doi: 10.1016/0306-3623(95)02050-0

15. Sarosiek J, Rourk RM, Piascik R, Namiot Z, Hetzel DP, McCallum RW. The effect of esophageal mechanical and chemical stimuli on salivary mucin secretion in healthy individuals. Am J Med Sci. 1994;308(1):23-31. doi: 10.1097/00000441-199407000-00006

16. Kejriwal S, Bhandary R, Thomas B, Kumari S. Estimation of levels of salivary mucin, amylase and total protein in gingivitis and chronic periodontitis patients. J Clin Diagn Res. 2014;8(10): ZC56-60. doi: 10.7860/JCDR/2014/8239.5042

17. Di Rienzo JA, Casanoves F, Balzarini MG, Gonzalez L, Tablada M, Robledo CW. InfoStat versión 2020. Argentina: Grupo InfoStat, FCA, Universidad Nacional de Córdoba; 2020. Disponible en: http://www.infostat.com.ar.

18. Perelman SB, Garibaldi LA, Tognetti PM. Experimentación y modelos Estadísticos, editor Facultad de Agronomía. Universidad de Buenos Aires; 2019. 475 p.

19. Sánchez GA, Miozza V, Delgado A, Busch L. Determinación de niveles salivales de mucina y amilasa en pacientes con periodontitis crónica. J Periodont Res. 2011;46(2):221-7. doi: 10.1111/j.16000765.2010.01332.x.

20. Acquier AB, PITA AKDC, Busch L, SÁNCHEZ GA. Comparison of salivary levels of mucin and amylase and their relation with clinical parameters obtained from patients with aggressive and chronic periodontal disease. J Appl Oral Sci. 2015;23(3):288-94. doi: 10.1590/1678-775720140458

21. Lundmark A, Johannsen G, Eriksson K, Kats A, Jansson L, Tervahartiala T, Rathnayake N, Åkerman S, Klinge B, Sorsa T, Yucel-Lindberg T. Mucin 4 and matrix metalloproteinase 7 as novel salivary biomarkers for periodontitis. J Clin Periodontol. 2017;44(3):247-54. doi: 10.1111/jcpe.12670 


\section{Nota de financiamiento:}

La fuente de financiamiento es la Universidad Nacional del Nordeste -Rep. Argentina.

\section{Nota declaración de interés:}

Los autores declaramos no tener conflicto de intereses de ningún tipo.

\section{Nota contribución de los autores:}

1. Concepción y diseño del estudio

2. Adquisición de datos

3. Análisis de datos

4. Discusión de los resultados

5. Redacción del manuscrito

6. Aprobación de la versión final del manuscrito

MJA ha contribuido en: $1,2,3,4,5$, y 6 .

RPJ ha contribuido en: $1,4,5$ y 6 .

\section{Nota de aceptación:}

Este artículo fue aprobado por la editora de la revista Mag. Dra. Vanesa Pereira-Prado. 